\title{
Pairwise correlation of genes involved in glucose metabolism: a potential diagnostic marker of cancer?
}

\author{
Meena Kishore Sakharkar ${ }^{1}$, Karthic Rajamanickam ${ }^{1}$, Shaoping Ji $^{2}$, Sarinder Kaur \\ Dhillon ${ }^{3}$ and Jian Yang ${ }^{1}$ \\ 1 Drug Discovery and Development Research Group, College of Pharmacy and Nutrition, University of Saskatchewan, \\ Saskatoon, SK S7N 5E5, Canada \\ ${ }^{2}$ Henan Provincial Engineering Centre for Tumor Molecular Medicine, Institute of Molecular Medicine, School of Basic Medical \\ Sciences, Henan University, Kaifeng, Henan Province 474004, P.R. of China \\ ${ }^{3}$ Institute of Biological Sciences, Faculty of Science, University of Malaya, Kuala Lumpur 50603, Malaysia \\ Correspondence to: Meena Kishore Sakharkar and Jian Yang, email: meena.sakharkar@usask.ca, jian.yang@usask.ca \\ Keywords: gene expression; gene pair correlation; diagnostic marker; pan-cancer analysis; protein-protein interaction network \\ Received: April 01, 2021 \\ Accepted: June 11, 2021 \\ Published: June 17, 2021
}

Copyright: ( 2021 Sakharkar et al. This is an open-access article distributed under the terms of the Creative Commons Attribution License (CC BY 3.0), which permits unrestricted use, distribution, and reproduction in any medium, provided the original author and source are credited.

\section{ABSTRACT}

Cancer is a highly malignant disease, killing approximately 10 million people worldwide in 2020. Cancer patient survival substantially relies on early diagnosis. In this study, we evaluated whether genes involved in glucose metabolism could be used as potential diagnostic markers for cancer. In total, 127 genes were examined for their gene expression levels and pairwise gene correlations. Genes $A D H 1 B$ and PDHA2 were differentially expressed in most of the 12 types of cancer and five pairs of genes exhibited consistent correlation changes (from strong correlations in normal controls to weak correlations in cancer patients) across all types of cancer. Thus, the two differentially expressed genes and five gene pairs could be potential diagnostic markers for cancer. Further preclinical and clinical studies are warranted to prove whether these genes and/or gene pairs would indeed aid in early diagnosis of cancer.

\section{INTRODUCTION}

Despite recent advances in diagnosis and treatment, cancer remains one of the top challenges to human health. Prognosis for advanced-stage and recurrent cancer remains poor. Cancer is highly heterogeneous and can remarkably reprogram metabolic pathways [1]. Metabolic reprogramming not only alters the type and concentration of intracellular and extracellular metabolites but also modulates gene expression and tumor microenvironment for cell proliferation and survival $[2,3]$. However, pancancer analysis of metabolic reprogramming is limited except for observation of increased glucose uptake and aerobic glycolysis (Warburg Effect) in cancer cells [4, 5].

Glucose is the major energy source for cells, and thus, glucose metabolism is essential for normal cell functions and survival. In cancer cells, glucose metabolism is opted to low-efficient aerobic glycolysis over oxidative phosphorylation. This is likely an evolutionary adaptation to the hypoxic microenvironment. Furthermore, abnormality in glucose metabolism, such as the Krebs cycle, can trigger cancer metastasis and resistance towards chemotherapy [6-9]. Although there is a dispute on whether aerobic glycolysis is the cause or consequence of cancer, it is clear that cancer usually initiates in a hypoxic region and the switch to aerobic glycolysis is far ahead of cancer being diagnosed by lab tests or medical imaging [10-12]. Therefore, analysis of gene expression in the glucose metabolic pathway not only provides us valuable information on carcinogenesis but also could be used for early diagnosis of cancer.

Early diagnosis remains crucial for cancer patient survival. Our previous study showed that gene expression correlation coefficient could be used as a prognostic/diagnostic biomarker for human breast cancer [13]. Recently, we also reported that loss of gene pair correlations in the sphingolipid metabolic pathway and tryptophan metabolic pathway could be a hallmark in cancer diagnosis $[14,15]$. In the current study, we undertake a pan-cancer analysis of gene expression and gene pair correlation for 127 genes involved in glucose metabolism using The Cancer Genome Atlas (TCGA). 

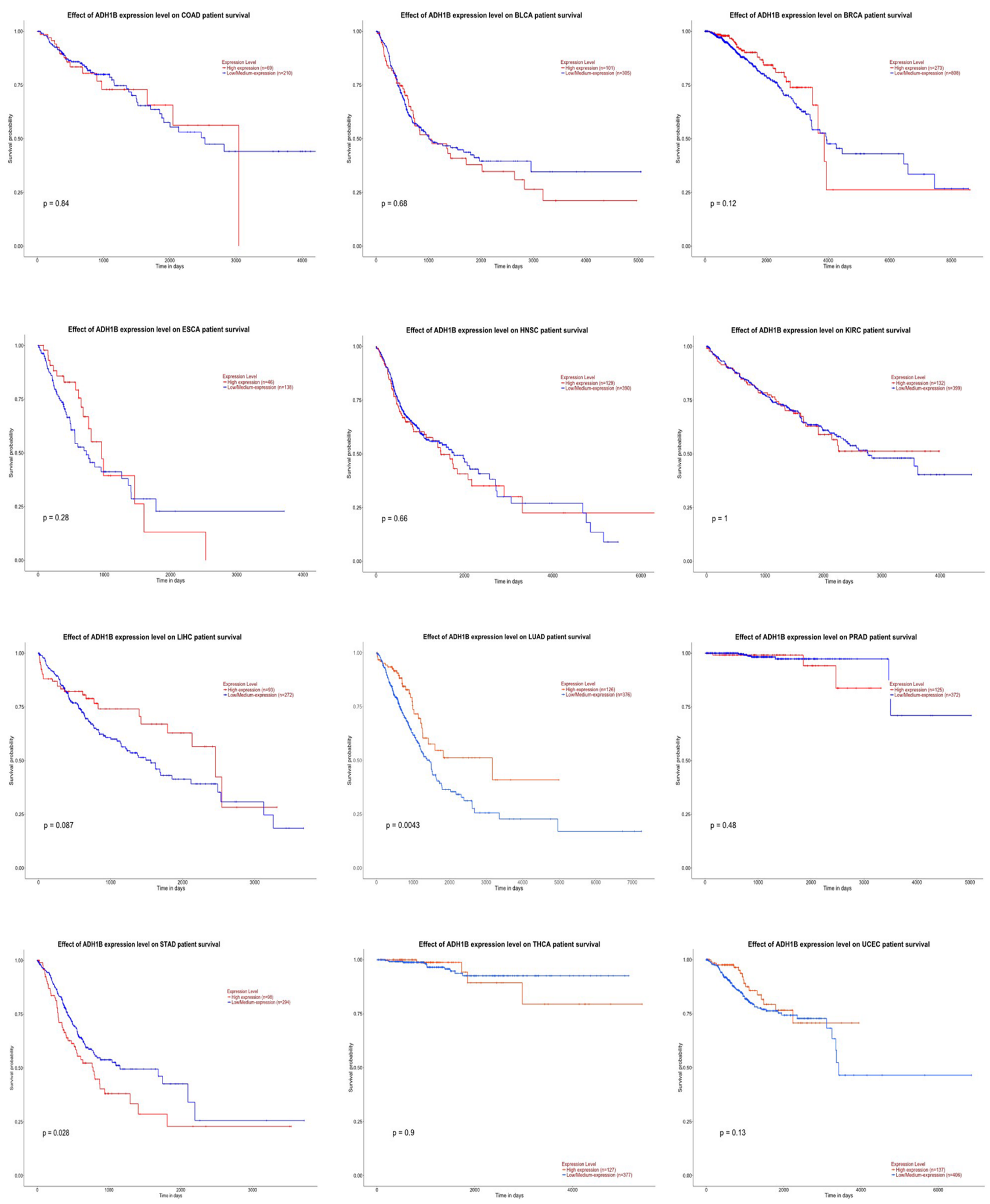

Figure 1: Kaplan-Meier plots of ADH1B in 12 different types of cancer. Patients with high expression of $A D H 1 B$ were shown in red and patients with low/medium expression of $A D H 1 B$ were shown in blue, respectively. $A D H 1 B$ is a favorable prognostic factor for lung adenocarcinoma (LUAD, $p=0.004$ ) but an unfavorable prognostic factor for stomach adenocarcinoma $(\mathrm{STAD}, p=0.028)$. The Kaplan-Meier plots were generated using online software UALCAN (http://ualcan.path.uab.edu, accessed on May 23, 2021). 
Table 1: Ten genes showing similar trend of expression change (either upregulated or downregulated) across all 12 types of cancer, with the two genes differentially expressed $\left(\left|\log _{2} \mathrm{FC}\right|\right.$ $\geq 1.00$ ) in most of the 12 types of cancer highlighted in red.

\begin{tabular}{|l|r|r|r|r|r|r|r|r|r|r|r|r|}
\hline Gene & BLCA & BRCA & COAD & ESCA & HNSC & KIRC & LIHC & LUAD & PRAD & STAD & THCA & UCEC \\
\hline ADH1B & -4.40 & -4.86 & -4.06 & -2.15 & -3.41 & -2.67 & -1.16 & -3.19 & -1.66 & -1.83 & -3.44 & -7.03 \\
\hline ALDH2 & -1.64 & -2.10 & -0.26 & -0.17 & -0.65 & -0.75 & -1.02 & -1.19 & -0.81 & -0.23 & -0.84 & -0.36 \\
\hline ENO1 & 0.96 & 0.33 & 1.00 & 1.48 & 1.14 & 0.64 & 1.27 & 1.22 & 0.19 & 1.12 & 0.17 & 1.53 \\
\hline EPM $2 A$ & -2.45 & -0.96 & -1.21 & -0.79 & -0.99 & -1.30 & -0.30 & -1.17 & -0.94 & -1.10 & -0.82 & -2.00 \\
\hline GAPDH & 0.67 & 1.05 & 0.90 & 1.29 & 0.56 & 1.50 & 1.35 & 1.72 & 0.08 & 0.64 & 0.44 & 1.45 \\
\hline LDHA & 0.62 & 0.56 & 0.72 & 1.50 & 1.31 & 1.71 & 0.05 & 1.36 & 0.42 & 0.85 & 0.07 & 1.38 \\
\hline MPC1 & -0.62 & -0.38 & -0.96 & -0.69 & -0.82 & -1.56 & -0.85 & -0.37 & -0.19 & -0.42 & -0.79 & -0.38 \\
\hline PDHA2 & N/A & 1.13 & 1.88 & 2.99 & 1.65 & 2.27 & 3.72 & 3.43 & 0.73 & 2.92 & 1.35 & 1.73 \\
\hline PFKFB4 & 2.39 & 1.26 & 0.84 & 1.15 & 1.65 & 2.45 & 2.43 & 0.81 & 0.21 & 0.11 & 0.18 & 2.33 \\
\hline PHKG2 & 0.71 & 1.12 & 0.48 & 0.95 & 1.01 & 0.28 & 0.86 & 0.86 & 0.26 & 0.96 & 0.31 & 1.01 \\
\hline
\end{tabular}

\section{RESULTS AND DISCUSSION}

Cancer is a heterogenous and complex disease, killing approximately 10 million people globally in 2020 [16]. Although recent advances, such as CAR T-cell therapy and immune checkpoint inhibitors, have provided more options for cancer treatment, prognosis for advanced-stage and recurrent cancer remains poor. For example, the 5-year survival rate for colon cancer drops from $92 \%$ for stage I down to $12 \%$ for stage IV in Canada [17]. Thus, early diagnosis is critical for patient's survival. Gene expression profiling using microarray and RNASeq data has been widely used to identify diagnostic or prognostic gene signatures, such as the 70-Gene Signature Assay, which are differentially expressed between cancer patients and normal controls. Moreover, these specific gene signatures may help in identifying drug design targets for cancer treatment.

Glucose metabolism is essential for normal cellular functions and cell growth, and switch to aerobic glycolysis (Warburg Effect) has been recognized as a characteristic of cancer. Thus, we undertook a pan-cancer analysis of 127 genes involved in glucose metabolism in 12 cancer datasets which meet the selection criteria of $\mathrm{N}_{\text {normal }} \geq$ 10 and $\mathrm{N}_{\text {cancer }} \geq 10$. The 12 types of cancer are bladder urothelial carcinoma (BLCA), breast invasive carcinoma (BRCA), colon adenocarcinoma (COAD), esophageal carcinoma (ESCA), head and neck squamous cell carcinoma (HNSC), kidney renal clear cell carcinoma (KIRC), liver hepatocellular carcinoma (LIHC), lung adenocarcinoma (LUAD), prostate adenocarcinoma
(PRAD), stomach adenocarcinoma (STAD), thyroid carcinoma (THCA), and uterine corpus endometrial carcinoma (UCEC). For each gene, the normalized $\log _{2} \mathrm{FC}$ in gene expression between cancer patients and normal controls was calculated and presented in Supplementary Table S1. As shown in Table 1, there were 10 genes showing similar trend of gene expression change (either upregulated or downregulated) across all 12 types of cancer. These genes are ADH1B, ALDH2, ENO1, EPM2A, GAPDH, LDHA, MPC1, PDHA2, PFKFB4 and PHKG2. However, only one gene, $A D H 1 B$ (highlighted in red in Table 1) was differentially downregulated in all types of cancer upon using $\left|\log _{2} \mathrm{FC}\right| \geq 1.00$ and $\mathrm{p}<0.05$ as the cut-off. This implicated that $A D H 1 B$ could be applied as a diagnostic marker for cancer. $A D H 1 B$ encodes alcohol dehydrogenase $1 \mathrm{~B}$, which catalyzes the oxidation of alcohol to form acetaldehyde. $A D H 1 B$ is downregulated in hepatocellular carcinoma [18] and its polymorphism is associated with increased risk for various types of cancer, such as colorectal cancer, gastric cancer, and esophageal cancer [19-21]. We further performed survival analysis and generated Kaplan-Meier plots for $A D H 1 B$ for the 12 types of cancer (Figure 1). $A D H 1 B$ is a favorable prognostic factor for lung adenocarcinoma (LUAD, $p=$ 0.004 ) but an unfavorable prognostic factor for stomach adenocarcinoma (STAD, $p=0.028$ ). Other than $A D H 1 B$, gene PDHA2 (also highlighted in red in Table 1) was differentially upregulated in most types of cancer except in bladder urothelial carcinoma (BLCA, data not available) and prostate adenocarcinoma $\left(\mathrm{PRAD}, \log _{2} \mathrm{FC}=0.73\right)$, implicating that $P D H A 2$ could also be used as a diagnostic 


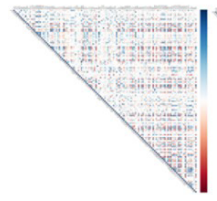

BLCA
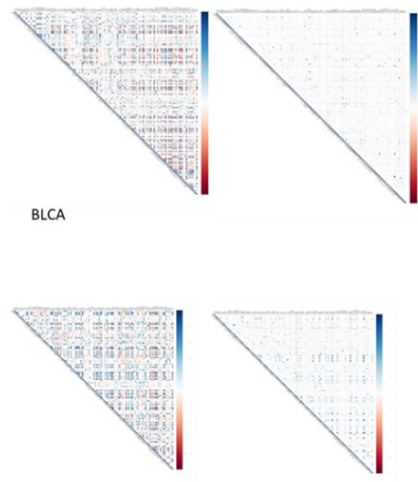

HNSC
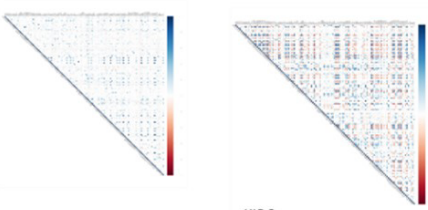

KIRC
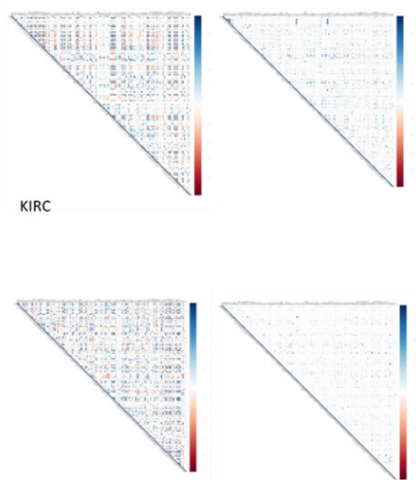

STAD
LHC
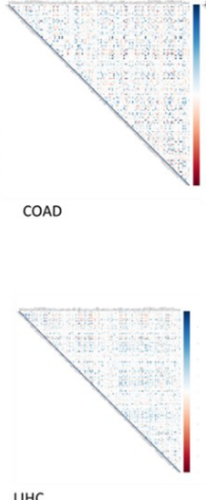

COAD

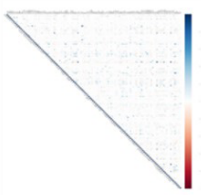

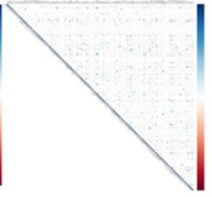
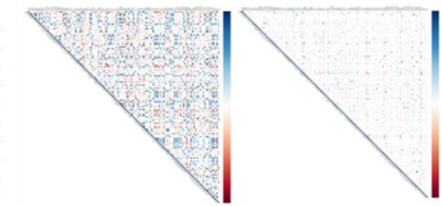

ESCA
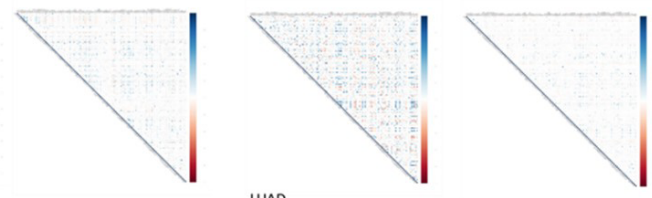

LUAD

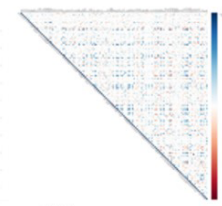

THCA
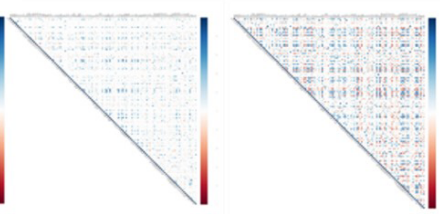

UCEC

Figure 2: Gene pair correlations of 127 genes involved in glucose metabolism across 12 different types of cancer (BLCA, BRCA, COAD, ESCA, HNSC, KIRC, LIHC, LUAD, PRAD, STAD, THCA and UCEC). Positive and negative correlations are represented by blue and red dots, respectively, and the sizes of the dots are proportional to the correlation coefficient values.

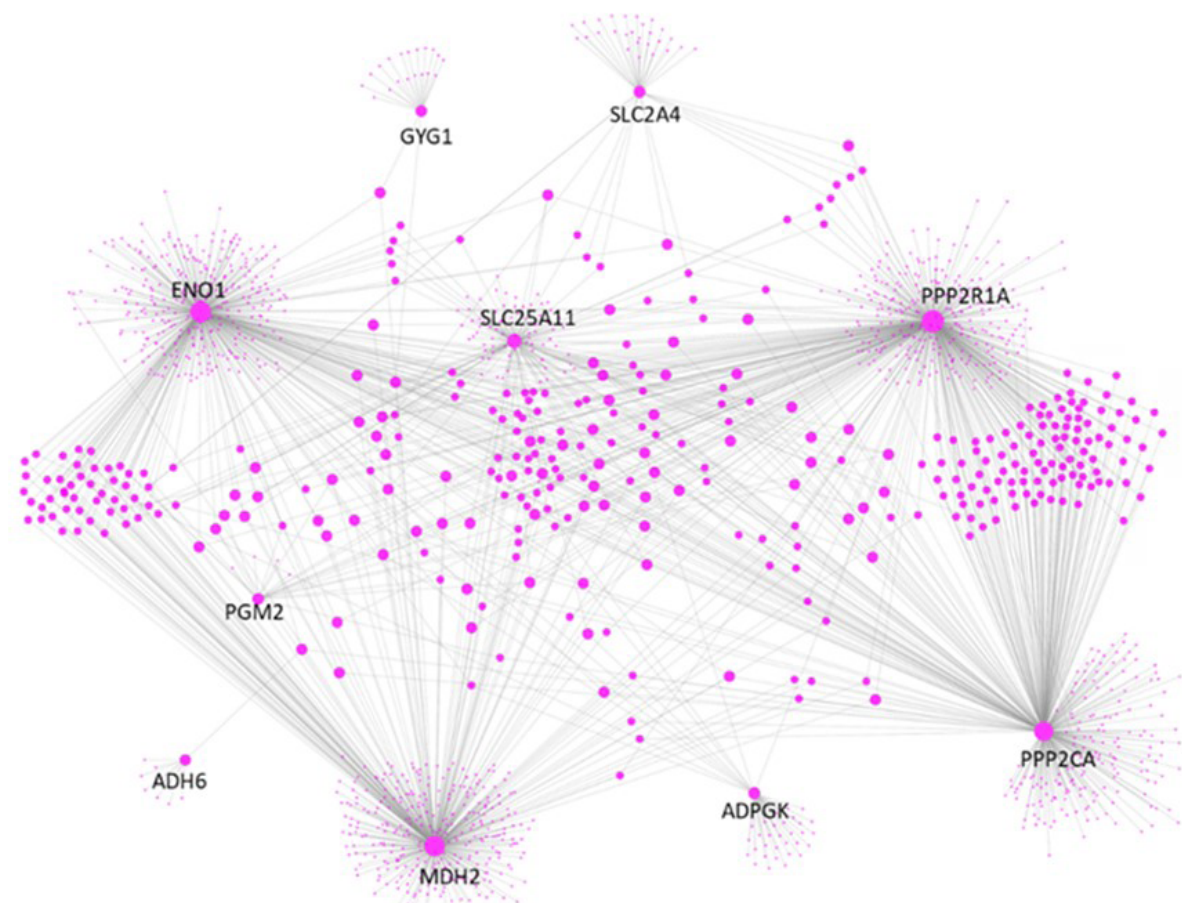

Figure 3: The protein-protein interaction (PPI) network for proteins encoded by the 5 pairs of genes with correlation changes consistent across all 12 types of cancer. 
Table 2: Five pairs of genes with correlation changes consistent across all 12 types of cancer.

\begin{tabular}{|l|l|}
\hline Gene pair & $\begin{array}{l}\text { Change of pairwise correlation from normal to } \\
\text { cancer }\end{array}$ \\
\hline$A D H 6-G Y C 1$ & Negative $\rightarrow$ Less negative \\
\hline$A D P G K-S L C 2 A 4$ & Negative $\rightarrow$ Less negative \\
\hline$E N O 1-P P P 2 R 1 A$ & Positive $\rightarrow$ Less positive \\
\hline$M D H 2-S L C 25 A 11$ & Positive $\rightarrow$ Less positive \\
\hline$P G M 2-P P P 2 C A$ & Positive $\rightarrow$ Less positive \\
\hline
\end{tabular}

marker. PDHA2 encodes subunit alpha 2 of pyruvate dehydrogenase $1 \mathrm{E}$, which is a major component of the pyruvate dehydrogenase complex (PDC) catalyzing the oxidative decarboxylation of pyruvate to form acetyl-CoA. $P D H A 2$ is predominately expressed in germ cells, whereas its homologue, PDHA1, is expressed in somatic cells [22]. It has been shown that downregulation of PDHA1 promotes cancer progression and acts as a poor prognostic factor for cancer [23-26]. However, the biological function of PDHA2 has barely been studied in cancer cells. Only recently, Lv et al. proposed gene pair PDHA2-APRT as a potential prognostic marker for breast cancer patients after treatment with tamoxifen [27].

Some gene signatures, especially single gene makers, have failed to serve as diagnostic or prognostic markers, because alterations of their expressions are not sufficient enough to be detected [28]. Carcinogenesis is a very complex process, which requires the coordination of multiple genes. Increased/decreased correlations among genes are highly likely happening prior to expression changes of the individual genes. Our previous studies have shown that pairwise correlation coefficients were dramatically decreased for genes involved in either sphingolipid metabolism or tryptophan metabolism in cancer patients as compared to normal controls $[14,15]$. Due to the crucial role glucose metabolism in normal cellular functions, we decided to calculate the pairwise gene correlation coefficients for the 127 genes involved in glucose metabolism. Glucose metabolism genes were widely and strongly correlated in normal controls, however, the gene-pair correlation coefficients were significantly decreased or lost in cancer patients for all 12 types of cancer (Figure 2). In addition, we examined whether any pair of genes exhibited consistent correlation change across all types of cancer in one of the following six correlation categories: positive $\rightarrow$ more positive, positive $\rightarrow$ less positive, positive $\rightarrow$ negative, negative $\rightarrow$ more negative, negative $\rightarrow$ less negative and negative $\rightarrow$ positive. Only 5 pairs of genes were identified (Table 2). Interestingly, the correlation became weaker for all 5 pairs of genes upon carcinogenesis, with $A D H 6-G Y C 1$ and $A D P G K-S L C 2 A 4$ changed from negatively correlated in normal controls to less negatively correlated in cancer patients and ENO1 - PPP2R1A, MDH2 - SLC25A11 and $P G M 2$ - PPP2CA changed from positively correlated in normal controls to less positively correlated in cancer patients. Thus, correlation coefficients for these 5 pairs of genes could be applied as a potential diagnostic maker for cancer and/or an indicator of cancer prevalence in a community when compared with a normal control. However, the differentially downregulated gene $A D H 1 B$ and differentially upregulated gene $P D H A 2$ were not present in the identified gene pairs. This implicates that genes may decouple even without significantly altering their respective expression level upon carcinogenesis. The decoupling of genes might help to diagnose cancer at a much earlier stage than the currently used diagnostic techniques, such as gene signature and cancer antigen assays, which depend on changes of gene or protein levels.

Finally, we constructed the PPI network for proteins encoded by the 5 pairs of genes. As illustrated in Figure 3 , the 10 proteins, especially ENO1, MDH2, PPP2R1A and PPP2CA, are hubs in the PPI network. They make 1513 direct interactions with other proteins, including 48 proteins involved in glucose metabolism. Because biological hubs are normally drug development targets, further studies are warranted to identify whether these 10 proteins could also be the intervention sites for cancer treatment.

\section{MATERIALS AND METHODS}

\section{Data acquisition}

A list of 127 genes involved in glucose metabolism was downloaded from PathCards (https://pathcards. genecards.org/), which is an integrated database of human biological pathways and their annotations. Cancer RNA-Seq datasets $\left(\mathrm{N}_{\text {normal }} \geq 10\right.$ and $\left.\mathrm{N}_{\text {cancer }} \geq 10\right)$ were downloaded from TCGA via the Genomic Data Commons (GDC) data portal. In total, 543 normal controls and 5641 cancer patients from 12 different types of cancer were 
Table 3: Numbers of normal controls and cancer patients in 12 different types of cancer.

\begin{tabular}{|l|l|l|l|}
\hline Type & Cancer description & Normal & Cancer \\
\hline BLCA & Bladder urothelial carcinoma & 19 & 414 \\
\hline BRCA & Breast invasive carcinoma & 113 & 1102 \\
\hline COAD & Colon adenocarcinoma & 41 & 471 \\
\hline ESCA & Esophageal carcinoma & 11 & 159 \\
\hline HNSC & Head and neck squamous cell carcinoma & 13 & 127 \\
\hline KIRC & Kidney renal clear cell carcinoma & 72 & 538 \\
\hline LIHC & Liver hepatocellular carcinoma & 50 & 371 \\
\hline LUAD & Lung adenocarcinoma & 59 & 533 \\
\hline PRAD & Prostate adenocarcinoma & 52 & 498 \\
\hline STAD & Stomach adenocarcinoma & 32 & 375 \\
\hline THCA & Thyroid carcinoma & 58 & 502 \\
\hline UCEC & Uterine corpus endometrial carcinoma & 23 & 551 \\
\hline
\end{tabular}

involved in the study. The numbers of cancer patients and normal controls for each type of cancer were summarized in Table 3. For each dataset, 60,483 RNA transcripts were analyzed in term of FPKM value.

\section{Identification and visualization of differentially expressed genes}

The protocol on identifying differentially expressed genes (DEGs) in cancer against normal using DEGseq in the $\mathrm{R}$ package has been published [14, 15]. Briefly, Likelihood Ratio Test (LRT) was applied, and sample expression profiles were screened using $p$-value $<0.05$. The output was expressed in normalized $\log _{2}$ fold-change ( $\left.\log _{2} \mathrm{FC}\right)$. Then, expression changes of the 127 genes involved in glucose metabolism were extracted for the 12 types of cancer (Supplementary Table S1).

\section{Computation and visualization of correlation matrix}

For each type of cancer, correlation matrix was calculated using cor function and visualized using corrplot function in the $\mathrm{R}$ package. Positive and negative correlations are represented in blue and red, respectively.

\section{Protein-protein interaction (PPI) network}

Human protein interactome (BIOGRIDORGANISM-Homo_sapiens-4.0.189.tab) was downloaded from the BioGRID database [29]. PPI data were then extracted from the protein interactome and plotted using Cytoscape [30] for genes involved in the 5 gene pairs which were conserved across all 12 types of cancer.

\section{CONCLUSION}

In this study, we evaluated the expression and gene pair correlation for 127 genes involved in glucose metabolism across 12 different types of cancer. Genes $A D H 1 B$ and $P D H A 2$ were differentially expressed in most of the 12 types of cancer. We also identified five pairs of genes having consistent correlation changes (weaker correlations in cancer patients) in all types of cancer. The two differentially expressed genes and five gene pairs could be potential diagnostic markers for cancer.

\section{Author contributions}

This research work was conceived and designed by MKS and JY and carried out by MKS, KR, SJ, SKD and JY. The manuscript was written by MKS and JY and approved by all authors.

\section{CONFLICTS OF INTEREST}

The authors declare no potential conflicts of interest.

\section{FUNDING}

This research was supported in part by a Discovery Grant (grant number: 417652) from the Natural Sciences and Engineering Research Council of Canada (M.K.S.), and a President's NSERC bridging grant (grant number: 419247) from the University of Saskatchewan (J.Y.). 


\section{REFERENCES}

1. Faubert B, Solmonson A, DeBerardinis RJ. Metabolic reprogramming and cancer progression. Science. 2020; 368:eaaw5473. https://doi.org/10.1126/science.aaw5473. PMID:32273439

2. Pavlova NN, Thompson CB. The Emerging Hallmarks of Cancer Metabolism. Cell Metab. 2016; 23:27-47. https:// doi.org/10.1016/j.cmet.2015.12.006. PMID:26771115

3. Xia L, Oyang L, Lin J, Tan S, Han Y, Wu N, Yi P, Tang L, Pan Q, Rao S, Liang J, Tang Y, Su M, et al. The cancer metabolic reprogramming and immune response. Mol Cancer. 2021; 20:28. https://doi.org/10.1186/s12943-02101316-8. PMID:33546704

4. Läsche M, Emons G, Gründker C. Shedding New Light on Cancer Metabolism: A Metabolic Tightrope Between Life and Death. Front Oncol. 2020; 10:409. https://doi. org/10.3389/fonc.2020.00409. PMID:32300553

5. Pascale RM, Calvisi DF, Simile MM, Feo CF, Feo F. The Warburg Effect 97 Years after Its Discovery. Cancers (Basel). 2020; 12:2819. https://doi.org/10.3390/ cancers12102819. PMID:33008042

6. Porporato PE, Payen VL, Pérez-Escuredo J, De Saedeleer CJ, Danhier P, Copetti T, Dhup S, Tardy M, Vazeille T, Bouzin C, Feron O, Michiels C, Gallez B, Sonveaux P. A mitochondrial switch promotes tumor metastasis. Cell Rep. 2014; 8:754-66. https://doi.org/10.1016/j. celrep.2014.06.043. PMID:25066121

7. Lu J, Tan M, Cai Q. The Warburg effect in tumor progression: mitochondrial oxidative metabolism as an anti-metastasis mechanism. Cancer Lett. 2015 (2 Pt A); 356:156-64. https://doi.org/10.1016/j.canlet.2014.04.001. PMID:24732809

8. McDonald OG, Li X, Saunders T, Tryggvadottir R, Mentch SJ, Warmoes MO, Word AE, Carrer A, Salz TH, Natsume S, Stauffer KM, Makohon-Moore A, Zhong Y, et al. Epigenomic reprogramming during pancreatic cancer progression links anabolic glucose metabolism to distant metastasis. Nat Genet. 2017; 49:367-76. https://doi. org/10.1038/ng.3753. PMID:28092686

9. Lin J, Xia L, Liang J, Han Y, Wang H, Oyang L, Tan S, Tian Y, Rao S, Chen X, Tang Y, Su M, Luo X, et al. The roles of glucose metabolic reprogramming in chemo- and radioresistance. J Exp Clin Cancer Res. 2019; 38:218. https://doi. org/10.1186/s13046-019-1214-z. PMID:31122265

10. Yu L, Chen X, Sun X, Wang L, Chen S. The Glycolytic Switch in Tumors: How Many Players Are Involved? J Cancer. 2017; 8:3430-40. https://doi.org/10.7150/ jca.21125. PMID:29151926

11. Cao L, Wu J, Qu X, Sheng J, Cui M, Liu S, Huang X, Xiang Y, Li B, Zhang X, Cui R. Glycometabolic rearrangements - aerobic glycolysis in pancreatic cancer: causes, characteristics and clinical applications. J Exp Clin
Cancer Res. 2020; 39:267. https://doi.org/10.1186/s13046020-01765-x. PMID:33256814

12. Danhier P, Bański P, Payen VL, Grasso D, Ippolito L, Sonveaux P, Porporato PE. Cancer metabolism in space and time: beyond the Warburg effect. Biochim Biophys Acta Bioenerg. 2017; 1858:556-72. https://doi.org/10.1016/j. bbabio.2017.02.001. PMID:28167100

13. Ling B, Chen L, Liu Q, Yang J. Gene expression correlation for cancer diagnosis: a pilot study. BioMed Res Int. 2014; 2014:253804. https://doi.org/10.1155/2014/253804. PMID:24818135

14. Sakharkar MK, Kaur Dhillon S, Chidambaram SB, Essa MM, Yang J. Gene Pair Correlation Coefficients in Sphingolipid Metabolic Pathway as a Potential Prognostic Biomarker for Breast Cancer. Cancers (Basel). 2020; 12:1747. https://doi.org/10.3390/cancers12071747. PMID:32630169

15. Sakharkar MK, Dhillon SK, Rajamanickam K, Heng B, Braidy N, Guillemin GJ, Yang J. Alteration in Gene Pair Correlations in Tryptophan Metabolism as a Hallmark in Cancer Diagnosis. Int J Tryptophan Res. 2020; 13:1178646920977013. https://doi. org/10.1177/1178646920977013. PMID:33354111

16. Siegel RL, Miller KD, Jemal A. Cancer statistics, 2020. CA Cancer J Clin. 2020; 70:7-30. https://doi.org/10.3322/ caac.21590. PMID:31912902

17. Survival statistics for colorectal cancer. Available online: https://www.cancer.ca/en/cancer-information/cancertype/colorectal/prognosis-and-survival/survival-statistics (accessed on 20-03-2021).

18. Liu X, Li T, Kong D, You H, Kong F, Tang R. Prognostic implications of alcohol dehydrogenases in hepatocellular carcinoma. BMC Cancer. 2020; 20:1204. https://doi. org/10.1186/s12885-020-07689-1. PMID:33287761

19. Guo XF, Wang J, Yu SJ, Song J, Ji MY, Zhang JX, Cao Z, Wang J, Dong WG. Meta-analysis of the ADH1B and ALDH2 polymorphisms and the risk of colorectal cancer in East Asians. Intern Med. 2013; 52:2693-99. https://doi. org/10.2169/internalmedicine.52.1202. PMID:24334570

20. Ishioka $\mathrm{K}$, Masaoka $\mathrm{H}$, Ito $\mathrm{H}$, Oze I, Ito S, Tajika M, Shimizu Y, Niwa Y, Nakamura S, Matsuo K. Association between ALDH2 and ADH1B polymorphisms, alcohol drinking and gastric cancer: a replication and mediation analysis. Gastric Cancer. 2018; 21:936-45. https://doi. org/10.1007/s10120-018-0823-0. PMID:29616362

21. Tan B, Ning N. Association of ADH1B Arg47His polymorphism with the risk of cancer: a meta-analysis. Biosci Rep. 2019; 39:BSR20181915. https://doi. org/10.1042/BSR20181915. PMID:30872408

22. Pinheiro A, Silva MJ, Graça I, Silva J, Sá R, Sousa M, Barros A, Tavares de Almeida I, Rivera I. Pyruvate dehydrogenase complex: mRNA and protein expression patterns of $\mathrm{E} 1 \alpha$ subunit genes in human spermatogenesis. 
Gene. 2012; 506:173-78. https://doi.org/10.1016/j. gene.2012.06.068. PMID:22750801

23. Liu L, Cao J, Zhao J, Li X, Suo Z, Li H. PDHA1 Gene Knockout In Human Esophageal Squamous Cancer Cells Resulted In Greater Warburg Effect And Aggressive Features In Vitro And In Vivo. OncoTargets Ther. 2019; 12:9899-913. https://doi.org/10.2147/OTT.S226851. PMID:31819487

24. Song L, Liu D, Zhang X, Zhu X, Lu X, Huang J, Yang L, $\mathrm{Wu}$ Y. Low expression of PDHA1 predicts poor prognosis in gastric cancer. Pathol Res Pract. 2019; 215:478-82. https://doi.org/10.1016/j.prp.2018.12.038. PMID:30611622

25. Chen TY, Hsieh YT, Huang JM, Liu CJ, Chuang LT, Huang PC, Kuo TY, Chia HY, Chou CY, Chang CW, Chen YF, Chen HM, Lo JF, Li WC. Determination of Pyruvate Metabolic Fates Modulates Head and Neck Tumorigenesis. Neoplasia. 2019; 21:641-52. https://doi.org/10.1016/j. neo.2019.04.007. PMID:31100640

26. Zhong Y, Li X, Ji Y, Li X, Li Y, Yu D, Yuan Y, Liu J, Li H, Zhang M, Ji Z, Fan D, Wen J, et al. Pyruvate dehydrogenase expression is negatively associated with cell stemness and worse clinical outcome in prostate cancers. Oncotarget. 2017; 8:13344-56. https://doi.org/10.18632/ oncotarget.14527. PMID:28076853

27. Lv F, Jin WH, Zhang XL, Wang ZR, Sun AJ. Tamoxifen therapy benefit predictive signature combining with prognostic signature in surgical-only ER-positive breast cancer. J Cell Physiol. 2019; 234:11140-48. https://doi. org/10.1002/jcp.27756. PMID:30537139

28. Goodison S, Sun Y, Urquidi V. Derivation of cancer diagnostic and prognostic signatures from gene expression data. Bioanalysis. 2010; 2:855-62. https://doi.org/10.4155/ bio.10.35. PMID:21083217

29. Oughtred R, Rust J, Chang C, Breitkreutz BJ, Stark C, Willems A, Boucher L, Leung G, Kolas N, Zhang F, Dolma $\mathrm{S}$, Coulombe-Huntington J, Chatr-Aryamontri A, et al. The BioGRID database: A comprehensive biomedical resource of curated protein, genetic, and chemical interactions. Protein Sci. 2021; 30:187-200. https://doi.org/10.1002/ pro.3978. PMID:33070389

30. Killcoyne S, Carter GW, Smith J, Boyle J. Cytoscape: a community-based framework for network modeling. Methods Mol Biol. 2009; 563:219-39. https://doi. org/10.1007/978-1-60761-175-2 12. PMID: 19597788 